\title{
Effects of Physical Activity on Liver Function in Patients with Non-alcoholic Fatty Liver Disease: A Meta-Analysis
}

\author{
Jingjing Li, Fan Wang, Kan Chen, Yujing Xia, Jie Lu, Yingqun Zhou, Chuanyong Guo* \\ Department of Gastroenterology, Shanghai Tenth People's Hospital, Tongji University School of Medicine, Shanghai 200072, China
}

Received: October 30, 2015; Accepted: December 02, 2015; Published: December 05, 2015

*Corresponding author: Chuanyong Guo, Department of gastroenterology, Shanghai Tenth People's Hospital, Tongji University of medicine, People's Republic of China, Tel: +86-21-66302535; Fax: +86-21-66303983; E-mail: guochuanyong@hotmail.com

\begin{abstract}
Absract NAFLD.

Aim: To evaluate the effects of physical exercise in patients with

Methods: We searched standard databases including PubMed, Cochrane Library, EMBASE, et. al. up to Feb. 2014 to obtain relevant literatures. The Standard Mean Differences (SMD) with 95\% confidence interval $(95 \% \mathrm{CI})$ was calculated to estimate the effects.

Results: ALT levels of patients before and after exercise so significantly reduced that liver function returned to positive (SMD $-0.40,95 \%$ CI $-0.75 \sim-0.05, P=0.03$ ). The significant differences observed between the experimental and control groups showed that exercise can effectively lower fasting blood glucose in patients with NAFLD (SMD - $0.33,95 \%$ CI - $0.64 \sim-0.02, P=0.04$ ).

Conclusion: Exercise did not change body weight, but did improve liver enzymes and blood glucose levels in patients diagnosed with non-alcoholic fatty liver disease.
\end{abstract}

Keywords: NAFLD; Physical exercise; Liver fibrosis; Hepatology

\section{Core Tip}

Non-Alcoholic Fatty Liver Disease (NAFLD) has been reported to be associated with the incidence of other chronic liver diseases, diabetes and atherosclerosis. However, lifestyle change has significance in the improvement and rehabilitation of patients. The aim of this meta-analysis was to evaluate the effects of physical exercise in patients with NAFLD.

\section{Introduction}

Non-Alcoholic Fatty Liver Disease (NAFLD) is a metabolic stress-induced liver disorder closely related to insulin resistance and genetic susceptibility. NAFLD is characterized by excessive fat deposition in liver cells and includes three types: Simple Fatty Liver (SFL), Non-Alcoholic Steatohepatitis (NASH) and its related cirrhosis [1]. According to the investigation by Lazo et al., the average prevalence rate of NAFLD in adults is approximately 10 to $30 \%$, and up to $25 \%$ of patients develop cirrhosis within a decade $[2,3]$. In Europe and other developed countries, NAFLD may affect the incidence of other chronic liver diseases, diabetes and atherosclerosis, and can lead directly to decompensate cirrhosis. NAFLD can endanger patients' health and lives [46]. Therefore, the identification of an effective intervention to improve patient quality of life has great significance.

However, most of the drugs administered for NAFLD, such as the cholesterol-lowering drugs, statins, and antioxidants, hepatoprotective drugs and insulin-sensitizing agents, have pharmacological toxicity resulting in side effects. In recent years, Oza, et al. [7], concluded that lifestyle changes including moderate aerobic exercise has significance in the improvement and rehabilitation of patients. Researchers worldwide have conducted a number of studies on the effects of exercise; however, large-scale trials have not been carried out due to limitations on the prevalence of NAFLD [8-11]. Therefore, in the present study we performed a meta-analysis of Randomized Controlled Trials (RCTs) to evaluate the effects of physical exercise in patients with NAFLD.

\section{Methods}

\section{Data sources and searches}

Relevant literature was obtained using standard databases including PubMed, Cochrane Library, EMBASE, CINAHL, Web of Science, and Science Citation Index (updated to Sep. 2015), and was limited to human studies published in English with a search strategy using the Medical Subject Headings (MeSH) and text key words"exercise", "non-alcoholic fatty liver disease", and "randomized controlled trial". We also performed a comprehensive manual search of all references from primary or review articles and abstracts to identify valuable studies.

\section{Study selection}

Studies included in this meta-analysis conformed to the following inclusion criteria:

1. Experimental design: RCTs on the effects of exercise in patients with NAFLD;

2. Experimental subject: patients with a diagnosis of NAFLD 
for more than one year identified according to the American Gastroenterological Association [12];

3. Experimental process: the study included a control group, moderate exercise intensity (exercise of more than $20 \mathrm{~min}$, more than twice a week), reported data on liver biochemical markers and

4. Subjects were excluded due to long-term alcohol consumption (more than $20 \mathrm{~g} / \mathrm{d}$ ).

\section{Data abstraction and quality assessment}

Data abstraction was independently performed by two investigators (Jingjing Li and Fan Wang) using a standardized data abstraction tool based on the Cochrane Handbook for Systematic Reviews of Intervention and any differences were resolved by consensus or by the third investigator (Kan Chen). The following information was extracted from each article: name of the first author, year of publication, sample size, exercise intensity and period, method of data collection and method used to deal with missing data, basic parameters (age, sex and body mass index, BMI) and liver biochemistry (alanine transaminase, ALT; aspartate aminotransferase, AST) before and after exercise, and adverse events. Exercise should be as aerobic.

Methodological quality of the studies was assessed by the Consolidated Standards of Reporting Trials (Consort statement) [13]. The studies included in the meta-analysis were evaluated using the 22-stem criteria for RCTs in order to maintain the high quality of the literature using scientific evaluation for RCT design. Each item received 1 score, and studies with a score of more than 18 were considered high quality. Two researchers evaluated the studies which were included in the meta-analysis when a consensus was reached. The meta-analysis was carried out according to the Cochrane Handbook of Systematic Reviews consistent with PRISMA guidelines [14].

\section{Statistical Analyses}

RevMan 5.2 (The Nordic Cochrane Centre, The Cochrane Collaboration, 2012) was used to analyze the extracted data. The Odds Ratio (OR) with 95\% Confidence Intervals (CI) for continuous data was evaluated. Dispersion extent was evaluated using the Standard Mean Difference (SMD). When the original literature did not report SMDs, they were assessed by other literature data. During assessment of data heterogeneity, when $\mathrm{P}$ values were $>0.10$ and an $\mathrm{I}^{2}$ value was $<50 \%$, the fixed effects model was used, otherwise a random effects model was used [15]. For in-depth study of the clinical heterogeneity of the results, we established a subgroup analysis as each study reported slightly different source data and target results. Data heterogeneity may lead to an exaggerated effect and if all blind inclusions lead to increased internal validity this could reduce the credibility of the analysis. We also used funnel plots to determine publication bias to increase data availability to the highest degree.

\section{Results}

\section{Study characteristics}

We searched 186 papers from the above-mentioned databases, and following strict selection, 5 papers were included in the study (Figure 1) $[8,9,11,16,17]$. These studies included patients with NAFLD enrolled in RCTs with a doubleblind physical exercise intervention and reported liver function indices including ALT and AST. The selected papers also included data on liver histopathology. The basic characteristics and quality scores of the selected studies are shown in Table 1. Patient age ranged from 15 to $60 y$ ears with a different number of patients in each study. Exercise intensity was moderate and mainly aerobic exercise. The duration of treatment in each study was different and ranged from 1 week to 6 months. According to Table 1 the duration of treatment ranged from 2 weeks to 6 months, and the results were used as an effective reference range.

\section{Meta-Analysis \\ BMI}

Five trials $[8,9,11,16,17]$ which included 187 patients reported changes in body weight using BMI as the standard. Heterogeneity $\left(P=0.01, I^{2}=69 \%\right)$ was high, but in the moderate range. Significant clinical heterogeneity was observed, however changes in the data may have been due to patient age and different BMIs. Therefore, the random effects model was used. No statistically significant difference in BMI was found in patients with NAFLD following exercise (SMD -0.17, 95\% CI - $0.75 \sim 0.41, P=0.57$; Figure 2).

\section{ALT level}

A total of four trials $[8,9,11,12]$ reported ALT level change before and after exercise. Analysis of the results using a fixed effects model synthesis was not statistically significant heterogeneity ( $P=0.33, I^{2}=12 \%$ ), and the description of the experimental results is homogeneous. We calculated the value of their combined effect to find that there was statistically significant between the experimental group and the control. The differences indicated that ALT levels of patients before and after exercise so significantly reduced that liver function returned to positive (SMD -0.40, 95\% CI -0.75 -0.05, $P=0.03$; Figure 3).

\section{Fasting glucose}

Nonalcoholic fatty liver disease is often seen in patients with diabetes due to metabolic disorders; therefore, we evaluated fasting blood glucose reported in four trials $[8,9,15,16]$ which helped us to recognize NAFLD as a standard indicator for glucose determination. We found that in 170 patients, fasting blood glucose testing showed no heterogeneity $\left(P=0.46, I^{2}=0 \%\right)$. The combined effect can be a better representation of the overall level. The significant differences observed between the experimental and control groups showed that exercise can effectively lower fasting blood glucose in patients with NAFLD (SMD -0.33, 95\% CI $-0.64 \sim-0.02, P=0.04$; Figure 4).

\section{Sensitivity Analyses}

Changes in blood glucose and ALT in patients with NAFLD suggested that the patient's condition improved following exercise; however, the significance of BMI requires further investigation. Re-validation of the five studies showed that 


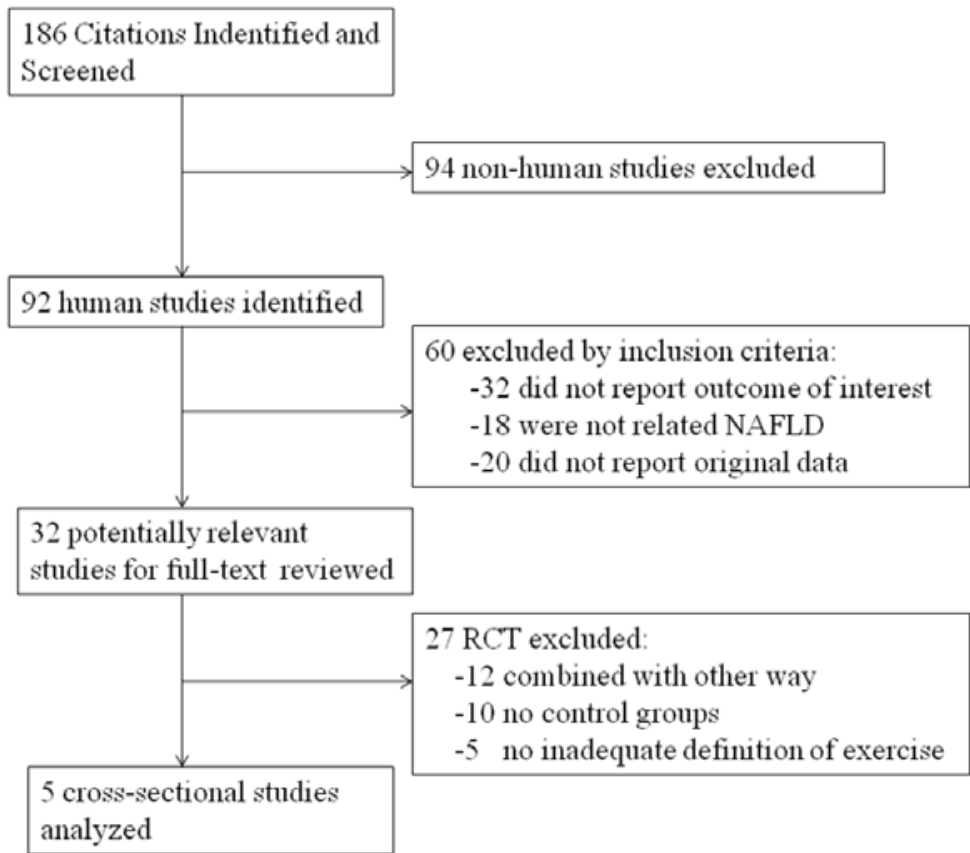

Figure 1: Trial identification, inclusion and exclusion.

Table 1: Characteristics of the studies included in the meta-analysis.

\begin{tabular}{|l|c|c|c|c|c|c|c|}
\hline Source & Score & $\begin{array}{c}\text { Age } \\
\text { (years) }\end{array}$ & $\begin{array}{c}\text { Sample } \\
\text { Size }\end{array}$ & $\begin{array}{c}\text { BMI } \\
\mathbf{k g} / \mathbf{m}^{2}\end{array}$ & $\begin{array}{c}\text { Fasting glucose } \\
\text { (mmol/L) }\end{array}$ & $\begin{array}{c}\text { ALT/AST } \\
\text { Duration of } \\
\text { treatment }\end{array}$ \\
\hline George, 2009[8] & 20 & 47 & 72 & 31.7 & 5.8 & $67.2 / 43.6$ & 3 months \\
\hline Eckard, 2013 [10] & 21 & 51 & 20 & 33.3 & 5.9 & $64.1 / 39.9$ & 6 months \\
\hline Sullivan, 2012 [11] & 19 & 58 & 18 & 35.2 & 6.2 & $39.7 /$ NR & 4 months \\
\hline Hallsworth, 2011 [16] & 20 & 57 & 21 & 32.3 & 6.3 & $60.6 /$ NR & 2 weeks \\
\hline Wang, 2008[17] & 20 & 15 & 57 & 28.5 & NR & $148.4 / 89.4$ & 1 month \\
\hline
\end{tabular}

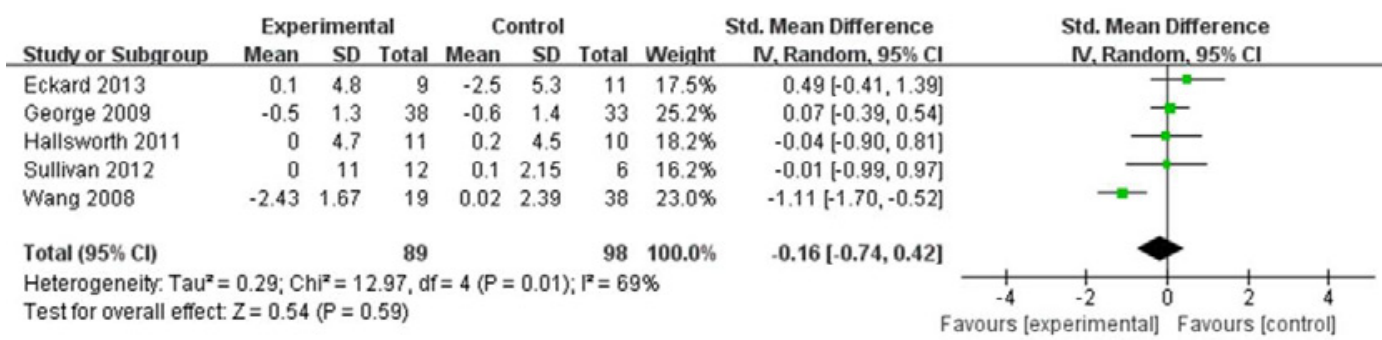

Figure 2: BMI in patients suffered with nonalcoholic fatty liver disease.

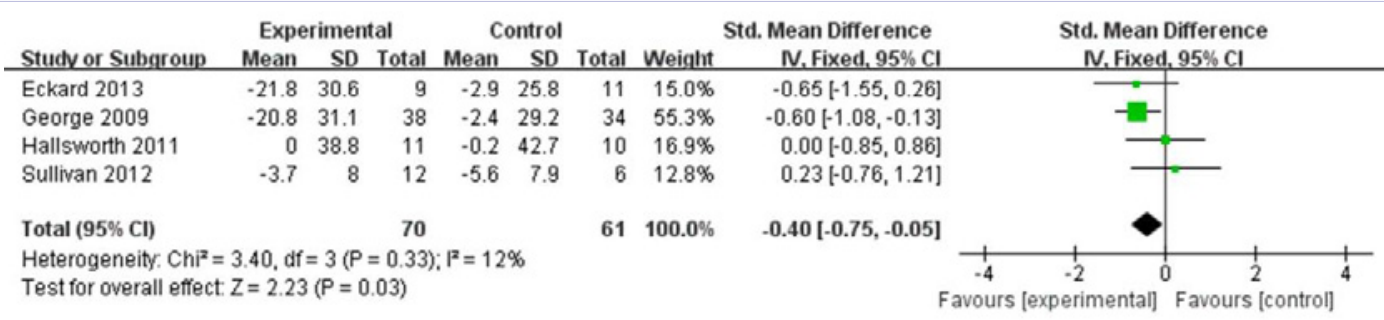

Figure 3: ALT level in patients with nonalcoholic fatty liver disease. 


\begin{tabular}{|c|c|c|c|c|c|c|c|c|c|}
\hline \multirow[b]{2}{*}{ Study or Subgroup } & \multicolumn{3}{|c|}{ Experimental } & \multicolumn{2}{|c|}{ Control } & \multirow[b]{2}{*}{ Total } & \multirow{2}{*}{ Weight } & \multirow{2}{*}{$\begin{array}{l}\text { Std. Mean Difference } \\
\text { IV, Fixed, } 95 \% \mathrm{CI}\end{array}$} & \multirow{2}{*}{$\begin{array}{l}\text { Std. Mean Difference } \\
\text { N. Fixed, } 95 \% \mathrm{Cl}\end{array}$} \\
\hline & Mean & SD & Total & Mean & SD & & & & \\
\hline Eckard 2013 & -0.5 & 0.8 & 9 & 0.13 & 0.54 & 11 & $11.1 \%$ & $-0.90[-1.84,0.03]$ & \\
\hline George 2009 & -0.2 & 0.3 & 38 & 0 & 0.8 & 34 & $44.5 \%$ & $-0.33[-0.80,0.13]$ & \\
\hline Hallsworth 2011 & -1.91 & 1.5 & 11 & 0.83 & 7.17 & 10 & $12.6 \%$ & $-0.52[-1.39,0.35]$ & \\
\hline Wang 2008 & -0.02 & 0.4 & 19 & 0 & 0.37 & 38 & $31.8 \%$ & $-0.05[-0.60,0.50]$ & \\
\hline Total $(95 \% \mathrm{Cl})$ & & & 77 & & & 93 & $100.0 \%$ & $-0.33[-0.64,-0.02]$ & \\
\hline \multicolumn{6}{|c|}{$\begin{array}{l}\text { Heterogeneity. } \text { Chi }^{2}=2.61, d f=3(P=0.46) ; I^{2}=0 \% \\
\text { Test for overall effect: } Z=2.09(P=0.04)\end{array}$} & & & & $\begin{array}{cccc}2 & -1 & 0 & 1 \\
\text { xperimental] } & \text { Favours [ }\end{array}$ \\
\hline
\end{tabular}

Figure 4: Fasting blood glucose in patients with nonalcoholic fatty liver disease.

patient age in one study was lower than that in the other studies, thus a meta-analysis of the results did not rule out inaccuracies. This study was excluded and the other four studies were analyzed again, and no change in BMI heterogeneity $\left(P=0.73, I^{2}=0 \%\right)$ was noted. These results indicated that the data were not statistically significant and were still consistent with the above-mentioned conclusions (SMD 0.12, 95\% CI $-0.47 \sim-0.7, P=0.7$ ).

\section{Publication Bias}

Funnel plot asymmetry of the meta-analysis results showed the presence of slight publication bias which was caused by the sample size and treatment effect (Figure 5).

\section{Discussion}

Due to improvements in living standards, fatty liver caused by obesity, diabetes, hyperlipidemia, and metabolic syndrome has increased and Non-Alcoholic Fatty Liver Disease (NAFLD) is a threat to human health. Worldwide research on this disease has significantly increased in the past few years and researchers are working to resolve this issue [18-21]. However, identification of the optimal treatment for NAFLD is a slow process due to the complexity of the disease. Many patients are faced with the transition from hepatitis to cirrhosis and even complete liver failure. Statins and insulin sensitizer therapy administered during this stage of the disease are ineffective [22]. Generic drugs with hepatic and renal toxicity can cause damage to other organs in addition to the liver. Eckard et al. [9], found that healthy lifestyle changes including diet, exercise, and daily vitamin supplements significantly improved liver function. However, some researchers believe that intensive exercise raises oxygen tolerance, in addition to increased consumption of oxygen $[23,24]$. Therefore, an anoxic environment is formed in the body and recovery of liver function is unfavorable. This study was performed to confirm existing research results, and included a large sample size to evaluate the effects of exercise on liver function and blood sugar levels.

\section{Description of research quality}

In this study, we conducted rigorous screening to ensure the inclusion criteria were fair. The selected randomized controlled trials were chosen due to their strict design and evaluation results. Two of these studies were small heterogeneous trials and assessment indicators met the requirements of the combined effect, thus can be combined in large sample sizes for statistical analysis to draw accurate conclusions. However, greater heterogeneity for BMI, analyzed by re-assessment methods, may have included age as a major factor causing these differences. Sensitivity analysis showed a low heterogeneity and a meaningful combined effect when one trial, which resulted in large differences but negative results, was excluded. Slight asymmetry in the funnel plots showed publication bias related to the sample size.

\section{Analysis of results}

Patients after a short period of moderate exercise showed no obvious bias in BMI which illustrated that weight loss was not obvious when a normal diet was consumed. Meaningful

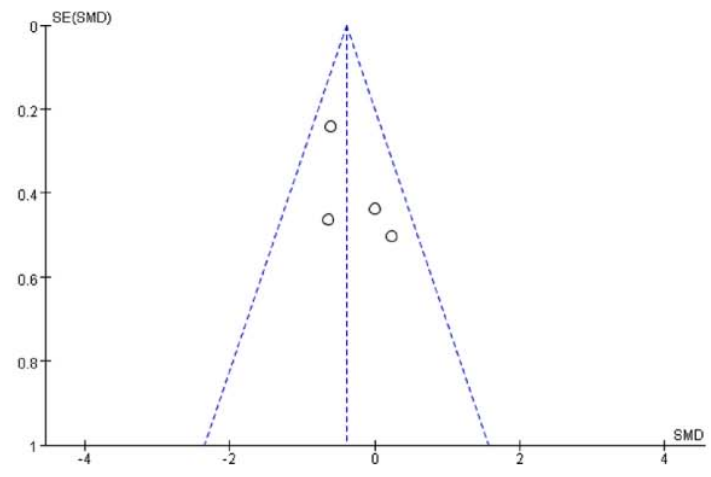

(a) ALT

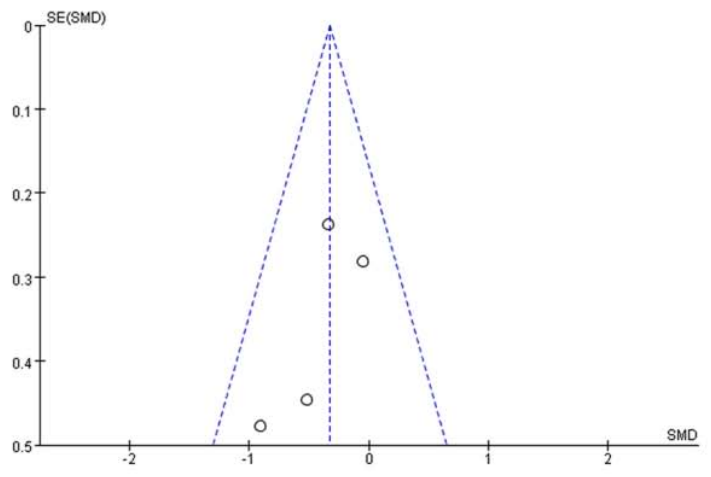

(b) Fasting blood glucose 
reductions in ALT and the glycemic index were observed Although this effect did not fully explain the improvements in liver function and blood lipid levels, the differences between the exercise group and the control group showed satisfactory changes to some extent. The main symptoms in NAFLD patients include bloating, gas and other liver disorders, and elevated blood sugar which can also lead to elevated blood lipids and a burden on the liver associated with treatment $[25,26]$. Delays in treatment can result in fat deposition in the liver. The present study found that exercise increased the activity of glycogen synthase, and affected sequestration of fatty acids and the glucose cycle in order to reduce the process of de novo lipogenesis [8,27]. Aerobic exercise stimulated the synthesis of triglycerides and reduced the deposition of fatty acid and its metabolites in the liver, and prevented the progression of liver fibrosis [11,28].

\section{Clinical significance}

Currently, the concept of a healthy lifestyle to minimize damage to the immune system due to drug therapy has great significance [29]. Exercise is widely performed by healthy individuals; however, most patients require bed rest and are unable to benefit from the positive effects of scientific and standardized exercise until they are well. Although this study did not explain the relationship between weight loss and improvement in related diseases, it is thought that exercise is closely related to disease. In clinical practice, professionals should actively promote a reasonable exercise program and urge patients to take part in a progressive exercise program to encourage the recovery of patients in an economical and effective way.

\section{Limitations of this study}

Although these five studies were high-quality randomized controlled trials, some limitations existed. For example: (1) Although we performed the meta-analysis to obtain a large sample size, the results were limited and further investigations are required; (2) It is difficult to achieve consistent baseline parameters due to differences in the populations studied, thus these differences should be compared to assess the effect on outcome. However, slight inaccuracies may exist due to the different measurement methods used. A subsequent study should standardize baseline parameters to determine the accurate effects of physical activity; and (3) the assessment of patients using non-uniform standards resulted in a few combined indicators which showed little improvement following exercise. In addition to the assessment of enzymatic and biochemical changes, personal feelings and psychological quality of life should also be used as evaluation criteria [30]. Therefore, SDS scores for depression and anxiety in patients should be included for a comprehensive evaluation of the extent of disease in patients.

In summary, exercise did not change body weight, but did improve liver enzymes and blood glucose levels in patients diagnosed with non-alcoholic fatty liver disease and indicated the significant importance of exercise in improving patients' health. Changes in oxygen resistance, lipid levels and liver enzyme levels improved liver histopathology and delayed disease progression to cirrhosis. Moreover, reduced cost and self-addressed exercise therapy, facilitated self-treatment of patients, to some extent. Of course, drug treatment is also necessary combined with a reasonable diet and moderate aerobic exercise to control weight, blood sugar, cholesterol and promote reversal of liver histopathology [31-34]. Therefore, we recommend that patients with NAFLD undergo regular aerobic exercise to reduce complications.

\section{Acknowledgements}

This study was supported by the Chinese Foundation for Hepatitis Prevention and Control (Grant nos. TQ20120005).

\section{Author Contributions}

Li JJ, Zhou YQ and Guo CY designed the research; Li JJ, Wang F, Chen K, Xia YJ and Lu J analyzed the data; Li JJ wrote the paper.

\section{Supportive Foundation}

This study was supported by the Chinese Foundation for Hepatitis Prevention and Control (Grant nos. TQ20120005).

\section{References}

1. Matteoni CA, Younossi ZM, Gramlich T, Boparai N, Liu YC, McCullough AJ. Nonalcoholic fatty liver disease: a spectrum of clinical and pathological severity. Gastroenterology. 1999;116(6):1413-9.

2. Harrison SA, Day CP. Benefits of lifestyle modification in NAFLD. Gut. 2007;56(12):1760-9.

3. Lazo M, Clark JM. The epidemiology of nonalcoholic fatty liver disease: a global perspective. Semin Liver Dis. 2008;28(4):339-50. doi: 10.1055/s0028-1091978.

4. Kim HJ, Kim HJ, Lee KE, Kim DJ, Kim SK, Ahn CW, et al. Metabolic significance of nonalcoholic fatty liver disease in nonobese, nondiabetic adults. Arch Intern Med. 2004;164(19):2169-75

5. Nyenwe EA, Williamson-Baddorf S, Waters B, Wan JY, Solomon SS. Nonalcoholic Fatty liver disease and metabolic syndrome in hypopituitary patients. Am J Med Sci. 2009;338(3):190-5. doi: 10.1097/ MAJ.0b013e3181a84bde.

6. Moore JB. Non-alcoholic fatty liver disease: the hepatic consequence of obesity and the metabolic syndrome. Proc Nutr Soc. 2010;69(2):211-20. doi: $10.1017 /$ S0029665110000030.

7. Oza N, Eguchi Y, Mizuta T, Ishibashi E, Kitajima Y, Horie H, et al. A pilot trial of body weight reduction for nonalcoholic fatty liver disease with a home-based lifestyle modification intervention delivered in collaboration with interdisciplinary medical staff. J Gastroenterol. 2009;44(12):1203-8. doi: 10.1007/s00535-009-0115-x.

8. St George A, Bauman A, Johnston A, Farrell G, Chey T, George J. Independent effects of physical activity in patients with nonalcoholic fatty liver disease. Hepatology. 2009;50(1):68-76. doi: 10.1002/hep.22940.

9. Eckard C, Cole R, Lockwood J, Torres DM, Williams CD, Shaw JC, et al. Prospective histopathologic evaluation of lifestyle modification in nonalcoholic fatty liver disease: a randomized trial. Therap Adv Gastroenterol. 2013;6(4):249-59. doi: 10.1177/1756283X13484078.

10. Promrat K, Kleiner DE, Niemeier HM, Jackvony E, Kearns M, Wands JR, et al. Randomized controlled trial testing the effects of weight loss on nonalcoholic steatohepatitis. Hepatology. 2010;51(1):121-9. doi: 10.1002/ hep.23276. 
11. Sullivan S, Kirk EP, Mittendorfer B, Patterson BW, Klein S. Randomized trial of exercise effect on intrahepatic triglyceride content and lipid kinetics in nonalcoholic fatty liver disease. Hepatology. 2012;55(6):1738-45. doi: 10.1002/hep.25548.

12. Chalasani N, Younossi Z, Lavine JE, Diehl AM, Brunt EM, Cusi K, et al. The diagnosis and management of non-alcoholic fatty liver disease: practice guideline by the American Gastroenterological Association, American Association for the Study of Liver Diseases, and American College of Gastroenterology. Gastroenterology. 2012;142(7):1592-609. doi: 10.1053/j. gastro.2012.04.001.

13. Lorette G, Maruani A. [The CONSORT statement (CONsolidated Standards of Reporting Trials)]. Ann Dermatol Venereol. 2013;140(67):431-5. doi: 10.1016/j.annder.2013.04.091.

14. Moher D, Liberati A, Tetzlaff J, Altman DG, PRISMA Group. Reprint-preferred reporting items for systematic reviews and meta-analyses: the PRISMA statement. Phys Ther. 2009 Sep;89(9):873-80.

15. Jackson D, Riley RD. A refined method for multivariate meta-analysis and meta-regression. Stat Med. 2014 Feb 20;33(5):541-54. doi: 10.1002/ sim.5957.

16. Wang CL, Liang L, Fu JF, Zou CC, Hong F, Xue JZ, et al. Effect of lifestyle intervention on non-alcoholic fatty liver disease in Chinese obese children. World J Gastroenterol. 2008;14(10):1598-602.

17. Hallsworth K, Fattakhova G, Hollingsworth KG, Thoma C, Moore S, Taylor R, et al. Resistance exercise reduces liver fat and its mediators in non-alcoholic fatty liver disease independent of weight loss. Gut. 2011;60(9):1278-83. doi: 10.1136/gut.2011.242073.

18. Wojcik M, Janus D, Dolezal-Oltarzewska K, Kalicka-Kasperczyk A, Poplawska K, Drozdz D, et al. A decrease in fasting FGF19 levels is associated with the development of non-alcoholic fatty liver disease in obese adolescents. J Pediatr Endocrinol Metab. 2012;25(11-12):1089-93. doi: 10.1515/jpem-2012-0253.

19. Chen S, Teoh NC, Chitturi S, Farrell GC. Coffee and non-alcoholic fatty liver disease: brewing evidence for hepatoprotection? J Gastroenterol Hepatol. 2014;29(3):435-41. doi: 10.1111/jgh.12422.

20. Cynis H, Kehlen A, Haegele M, Hoffmann T, Heiser U, Fujii M, et al Inhibition of Glutaminyl Cyclases alleviates CCL2-mediated inflammation of non-alcoholic fatty liver disease in mice. Int J Exp Pathol. 2013 Jun;94(3):217-25. doi: 10.1111/iep.12020.

21. Rodriguez A, Gena P, Mendez-Gimenez L, Rosito A, Valenti V, Rotellar F, et al. Reduced hepatic aquaporin- 9 and glycerol permeability are related to insulin resistance in non-alcoholic fatty liver disease. Int J Obes (Lond). 2014;38(9):1213-20. doi: 10.1038/ijo.2013.234.

22. Eslami L, Merat S, Malekzadeh R, Nasseri-Moghaddam S, Aramin H. Statins for non-alcoholic fatty liver disease and non-alcoholic steatohepatitis. Cochrane Database Syst Rev. 2013;12:CD008623. doi: 10.1002/14651858.CD008623.
23. Kojima H, Sakurai S, Uemura M, Fukui H, Morimoto H, Tamagawa Y. Mitochondrial abnormality and oxidative stress in nonalcoholic steatohepatitis. Alcohol Clin Exp Res. 2007;S61-6.

24. Xuan Zou, Chunhong Yan, Yujie Shi, Ke Cao, Jie Xu, Xun Wang et al. Mitochondrial Dysfunction in Obesity-Associated Nonalcoholic Fatty Liver Disease: The Protective Effects of Pomegranate with Its Active Component Punicalagin. Antioxid Redox Signal. 2014;21(11):1557-1570. doi: $10.1089 /$ ars.2013.5538.

25. Perseghin G, Price TB, Petersen KF, Roden M, Cline GW, Gerow K, et al. Increased glucose transport-phosphorylation and muscle glycogen synthesis after exercise training in insulin-resistant subjects. N Engl J Med. 1996;335(18):1357-62.

26.Zou CC, Liang L, Hong F, Fu JF, Zhao ZY. Serum adiponectin, resistin levels and non-alcoholic fatty liver disease in obese children. Endocr J. 2005;52(5):519-24.

27. Wong VW, Chan HL. Should all patients with nonalcoholic fatty liver disease undergo oral glucose tolerance test? J Gastroenterol Hepatol. 2011;26(3):419-20. doi: 10.1111/j.1440-1746.2010.06594.x.

28. Qureshi K, Clements RH, Saeed F, Abrams GA. Comparative evaluation of whole body and hepatic insulin resistance using indices from oral glucose tolerance test in morbidly obese subjects with nonalcoholic Fatty liver disease. J Obes. 2010;2010. pii: 741521. doi: 10.1155/2010/741521.

29. Suzuki A, Lindor K, St SJ, Lymp J, Mendes F, Muto A, et al. Effect of changes on body weight and lifestyle in nonalcoholic fatty liver disease. J Hepatol. 2005;43(6):1060-6.

30. Liu WY, Lu DJ, Du XM, Sun JQ, Ge J, Wang RW, et al. Effect of aerobic exercise and low carbohydrate diet on pre-diabetic non-alcoholic fatty liver disease in postmenopausal women and middle aged men--the role of gut microbiota composition: study protocol for the AELC randomized controlled trial. BMC Public Health. 2014;14:48. doi: 10.1186/1471-2458$14-48$.

31. Mishra S, Yadav D, Gupta M, Mishra H, Sharma P. A Study of Carotid Atherosclerosis in Patients with Non-alcoholic Fatty Liver Disease. Indian J Clin Biochem. 2013;28(1):79-83. doi: 10.1007/s12291-012-0286-8.

32. Chen S, Zhou H, Lin M, Mi R, Li L. Decoction vs extracts-mixed solution: effect of yiqihuoxue formula on non-alcoholic fatty liver disease in rats. J Tradit Chin Med. 2013;33(5):513-7.

33. Fan JG, Cao HX. Role of diet and nutritional management in non-alcoholic fatty liver disease. J Gastroenterol Hepatol. 2013;28(4):81-7. doi: 10.1111/ jgh. 12244

34. Alisi A, Ceccarelli S, Nobili V. Transient elastography and serum biomarkers: two-step screening methods for liver fibrosis in nonalcoholic fatty liver disease before liver biopsy. Expert Opin Med Diagn. 2012;6(5):377-80. doi: 10.1517/17530059.2012.713343. 